\title{
Excited-State Positronium Formation from Helium, Argon, and Xenon
}

\author{
D. J. Murtagh, D. A. Cooke, and G. Laricchia \\ UCL Department of Physics and Astronomy, University College London, Gower Street, London WC1E 6BT, United Kingdom
}

(Received 19 December 2008; published 1 April 2009)

\begin{abstract}
The cross sections for the formation of positronium in the $2 P$ state in collisions of positrons with $\mathrm{He}$, Ar, and Xe atoms have been determined by measuring coincidences between the remnant ion and the Lyman- $\alpha$ photon from positronium. The maximum fractional contributions of these to the total Ps formation cross sections increase from approximately $0.06 \pm 0.01$ in He to $0.12 \pm 0.04$ in $\mathrm{Ar}$ and $0.26 \pm$ 0.09 in Xe. In the case of He, good agreement is found with a coupled-state calculation; for Ar and Xe, measurements are compared with a distorted-wave Born approximation.
\end{abstract}

PACS numbers: 34.80.Uv, 36.10.Dr

Positronium (Ps) is the quasistable bound state of a positron $\left(e^{+}\right)$and an electron $\left(e^{-}\right)$. It is readily formed in encounters of positrons with matter, and its own interactions with atoms and molecules (e.g., [1]), including its fragmentation [2,3] and even its combination with another Ps atom [4], are amenable to experimental investigations. Interest in positronium encompasses the quest for the understanding of fundamental matter-antimatter interactions (e.g., [5-7]), tests of collision physics theories (e.g., $[8,9]$ ) and bound-state QED calculations (e.g., [10]), the analysis of energetic events occurring in the Galactic center (where it is estimated 93\% of all annihilations occur through the decay of Ps) [11], and problems of medical relevance [e.g. [12]].

Positronium is structurally equivalent to a hydrogen atom with half the reduced mass and thus Bohr energy levels $\left(E_{n}=-6.8 \mathrm{eV} / n^{2}\right)$. Its decay into $m \gamma$ rays is governed by $C P$ conservation according to $(-1)^{m}=$ $(-1)^{L+S}$, where $L$ and $S$ are the Ps orbital and spin angular momenta, respectively. Thus ground-state para- $\left(1^{1} S_{0}\right)$ and ortho- $\left(1^{3} S_{1}\right)$ positronium decay dominantly into 2 and 3 $\gamma$ rays, respectively, with corresponding lifetimes $\tau_{\gamma} \approx$ 125 ps and 143 ns. Those of the excited $S$ states increase with $n^{3}$, while states with $L>0$ are considerably longer lived due to their wave functions having a node at the origin, resulting in $\tau_{\gamma}(2 P)$ being extended to (0.1-3) ms and the decay of $2 P$ states being dominated by Lyman- $\alpha$ transitions ( $\tau_{\alpha} \approx 3.2 \mathrm{~ns}$ ). Conversely, $2 S \rightarrow 1 S$ transitions by the emission of a single photon are forbidden, and, in the absence of perturbative forces, the intrinsic survival of the metastable $2 S$ state is limited by annihilation.

The first unambiguous observation of excited-state positronium ( $\left.\mathrm{Ps}^{*}\right)$ was in the $2 P$ state [13]. This was achieved by bombarding a room temperature Ge target with a slow positron beam $(E=25$ and $40 \mathrm{eV})$ and by measuring the coincidences between an annihilation $\gamma$ ray and the Lyman- $\alpha$ deexcitation photon $(\lambda=243 \mathrm{~nm})$. A decade later, using a similar method, $\mathrm{Ps}^{*}$ was also observed in low density gases (namely, $\mathrm{Ne}, \mathrm{Ar}$, and $\mathrm{H}_{2}$ ) formed by positron projectiles of a few tens of eV [14]. While formation cross sections for Ps in the $2 S$ and $2 P$ states have been theoretically calculated for H (e.g., [15]), He [16-20], and the noble gases [21], experimental determinations have been lacking until now. It was recently considered that structure observed in the total positronium formation cross section $\left(Q_{\mathrm{Ps}}\right)$ for the heavier noble gases might be due to Ps* $^{*}$ [22-24], and estimates were made which inferred increasing contributions from Ps* to $Q_{\mathrm{Ps}}$ as the target atomic number was increased from $\mathrm{He}$ to $\mathrm{Xe}$ [22]. However, discrepancies exist on the magnitude [25] and the conjectured physical origin $[24,26]$ of this structure.

In the present work, coincidences between the residual ion and the deexcitation photon from Ps $^{*}$ have been measured in order to determine $Q_{\mathrm{Ps}^{*}}$ for the first time. In comparison with the previous work [14], the present method has the advantage that, by detecting ions rather than annihilation quanta, the signal can be easily enhanced by focusing onto a detector and a number of now-wellknown systematic effects [27] are avoided. Helium, argon, and xenon targets have been chosen to test available theories as well as the hypothesis of Ref. [22].

The experimental apparatus generating the beam of low energy positrons $(\Delta E \sim 2 \mathrm{eV})$ has been described in detail elsewhere $[28,29]$. Briefly, fast $\beta^{+}$emitted from a ${ }^{22} \mathrm{Na}$ source are moderated by annealed $W$ meshes. The slow positrons are radially confined by a magnetic field $(\vec{B} \approx$ $100 \mathrm{G})$ along the length of the beam line and pass through a bent solenoid, an electron repeller, and a Wien filter in order to reduce the number of unwanted $\gamma$ rays, secondary electrons, and fast positrons transported to the interaction region. A positively biased electrode may be used to repel the slow portion of the beam, allowing measurement of the background produced by the remaining particles. The interaction region is a hemispherical gas cell constructed from polished Al. A small electrostatic lens held at $-500 \mathrm{~V}$ extracts ions from the cell towards the detector, consisting of a channel electron multiplier (CEM) housed in a separately pumped chamber. A photomultiplier tube (PMT) with sensitivity in the range $200 \mathrm{~nm} \leq \lambda \leq$ $600 \mathrm{~nm}$ is mounted on an extension arm in order to remove it from the beam-guiding magnetic field. The extension arm is lined with UV reflectors, consisting of glass tubes 
coated with $\mathrm{Al}+\mathrm{MgF}_{2}$. A removable borosilicate glass disk may be inserted in front of the PMT to reduce its sensitivity range to $280 \mathrm{~nm} \leq \lambda \leq 600 \mathrm{~nm}$. Measurements of ion-photon coincidences are performed with and without the glass filter. The difference between the two measurements therefore corresponds to the detection of photons with wavelengths of $200 \mathrm{~nm} \leq \lambda \leq 280 \mathrm{~nm}$. The positron beam intensity is monitored by another CEM at the end of the flight path. Two ${ }^{22} \mathrm{Na}$ sources were used $(\sim 5$ and $50 \mathrm{mCi})$; for $\mathrm{He}$ and $\mathrm{Ar}$, the measured beam intensity was typically $\sim 10^{3} \mathrm{~s}^{-1}$, whereas for $\mathrm{Xe}$, the stronger source yielded $\sim 10^{4} \mathrm{~s}^{-1}$.

The total ion yield $\left(Y_{i}\right)$ is given by

$$
Y_{i}(E)=\frac{N_{i}-B_{i}}{N_{+}-B_{+}},
$$

where $N_{i}$ and $N_{+}$refer, respectively, to the ion and incident beam rates and $B_{i,+}$ represent the associated backgrounds measured by biasing off the slow portion of the beam. $Y_{i}$ is directly proportional to the total ionization cross section:

$$
Q_{i}^{t}=\sum Q_{x}=\frac{1}{\rho l_{\mathrm{eff}}} \frac{\epsilon_{+}}{\epsilon_{i}} Y_{i},
$$

where $Q_{x}$ is the cross section for any ion-producing process, i.e., Ps formation, direct ionization, annihilation, etc. (e.g., [30]), $\rho$ is the target number density, $l_{\text {eff }}$ is the effective length of the cell, $\epsilon_{+}$is the detection efficiency for positrons, and $\epsilon_{i}$ that for the ions, the latter comprising that for extraction.

The yield of Ps* is accordingly given by

$$
Y_{\mathrm{Ps}^{*}}=\frac{\left(N_{\mathrm{go}}-B_{\mathrm{go}}\right)-\left(N_{\mathrm{gi}}-B_{\mathrm{gi}}\right)}{N_{+}-B_{+}},
$$

where $N_{\mathrm{go}}$ is the coincidence rate obtained without the glass filter and by integrating the ion time-of-flight (TOF) spectra over the region corresponding to the desired charge-to-mass ratio, $N_{\mathrm{gi}}$ that with the filter inserted, and $B_{\mathrm{go}, \mathrm{gi}}$ the associated random backgrounds determined from a flat portion of the TOF spectra. The beam-off backgrounds were found to be negligible for the coincidence measurements.

The cross section for the formation of excited-state positronium $Q_{\mathrm{Ps}^{*}}$ is then determined according to

$$
Q_{\mathrm{Ps}^{*}}=\left(\frac{1}{\rho l_{\mathrm{eff}}} \frac{\epsilon_{+}}{\epsilon_{i}}\right)\left(\frac{1}{C_{r}} \frac{4 \pi}{\Delta \Omega}\right)\left(\frac{1}{\epsilon_{\mathrm{PMT}}}\right) Y_{\mathrm{Ps}^{*}},
$$

where $\Delta \Omega=(0.0164 \pm 0.0004)$ sr is the solid angle subtended by the PMT; $C_{R}=(2.40 \pm 0.22)$ corresponds to the enhancement of the photon collection due to the UV reflectors (determined by performing measurements without the light guides and the cell blackened with graphite), and $\epsilon_{\mathrm{PMT}}$ is the quantum efficiency of the PMT [ $\sim(20 \pm$ 2)\% at $\lambda<280 \mathrm{~nm}$ ] (Electron Tubes LTD.). $Y_{i}$ is measured simultaneously and allows the determination of the first term using Eq. (2) and $Q_{i}^{t}$ from Ref. [29] for He and Ref. [22] for Ar and Xe.
Contributions to the present measurements from Ps states other than the $2 P$ have been considered and will be discussed in detail elsewhere [31]. Briefly, they may originate from perturbations of the metastable $2 S$ state or from states with $n>2$. Lyman- $\alpha$ deexcitation from the $2 S$ state may occur via Stark mixing or collisional deexcitation. The former may be induced by the static electric field from the ion extraction lens and by the motion of the Ps transversely to the guiding magnetic field [32]. Collisional deexcitation of the $2 S$ via $2 P$ state has been evaluated using the cross section recently calculated in Refs. $[33,34]$ together with a Bohr-scaled total cross section, i.e., $Q_{t} \propto n^{4}$. In combination, these effects imply a contribution from the $2 S$ state to the measured signal in the range of $\sim(1-3) \%$. Using an isotropic distribution instead of the shape of the differential cross section for ground-state Ps formation [35] to estimate the average Ps* velocity perpendicular to the $B$ field, the overall contribution from Stark-quenched $\operatorname{Ps}(2 S)$ increases by less than $1 \%$.

The possibility of detection of states with $n>2$, either directly (i.e., $3 P \rightarrow 1 S$ ) or via cascade from $n>2$ via $2 P$ (e.g., $3 D \rightarrow 2 P$ ) has been estimated by calculating the deexcitation probability before a collision occurs (assumed for $n>2$ to result in breakup with unit probability, as in the case of $\mathrm{H}$ scattering $[36,37]$ ) and weighting this by $1 / n^{3}$, taken as a measure of the formation probability into higher $n$ states $[19,24]$. Overall, the contribution arising from states with $n>2$ is assessed to be again of the order of a few percent. Decreasing the probability of fragmentation of $\operatorname{Ps}(n>2)$ from unity to $50 \%$ [as is the case for $\operatorname{Ps}(n=$ 1)] (e.g., [30]) gives a contribution of $<5 \%$ for all targets. Thus states other than $2 P$ are expected to contribute $<10 \%$ to the present measurement. Henceforth, the measured cross section shall be referred to as $Q_{\mathrm{Ps}}(2 P)$.

The results are presented in Figs. 1-3 for $\mathrm{He}, \mathrm{Ar}$, and $\mathrm{Xe}$, respectively. The error bars shown in these figures comprise the statistical errors on the yields and the $\pm 14 \%$ overall uncertainty on the normalization factors in Eq. (4). Above each figure is the percentage contribution to the total (all $n$ ) $Q_{\mathrm{Ps}}$ (shown in the inset) [22,29]. In the case of $\mathrm{He}, Q_{\mathrm{Ps}}(2 P)$ rises from the threshold at $22.9 \mathrm{eV}$ reaching a maximum of $(2.6 \pm 0.6) \times 10^{-18} \mathrm{~cm}^{2}$ at an energy around $40 \mathrm{eV}$. The cross section decays rapidly after $50 \mathrm{eV}$ towards zero (within errors) at $100 \mathrm{eV}$. The maximum contribution to $Q_{\mathrm{Ps}}($ all $n$ ) [29] may be seen in the figure to be $(6 \pm 1) \%$. For $\operatorname{Ar}, Q_{\mathrm{Ps}}(2 P)$ increases from the threshold at $14.1 \mathrm{eV}$ and peaks near $40 \mathrm{eV}$ with a magnitude of $(2.2 \pm 0.5) \times 10^{-17} \mathrm{~cm}^{2}$, reducing to zero within errors by $140 \mathrm{eV}$. In this case, the maximum contribution to $Q_{\mathrm{PS}}$ is $(12 \pm 4) \%$. Finally, in the case of $\mathrm{Xe}$, the experimental $Q_{\mathrm{Ps}}(2 P)$ rises from the threshold at $10.4 \mathrm{eV}$ and peaks at approximately $23 \mathrm{eV}$ with a magnitude of $(1.4 \pm 0.5) \times 10^{-16} \mathrm{~cm}^{2}$ decaying rapidly after $25 \mathrm{eV}$. For $\mathrm{Xe}, \operatorname{Ps}(2 P)$ contributes a maximum of $(26 \pm 9) \%$ to $Q_{\mathrm{Ps}}$ [22]. Note that, for Ar and Xe, the uncertainties on the percentage contributions for the higher energy points are too large for the values to be meaningful. 


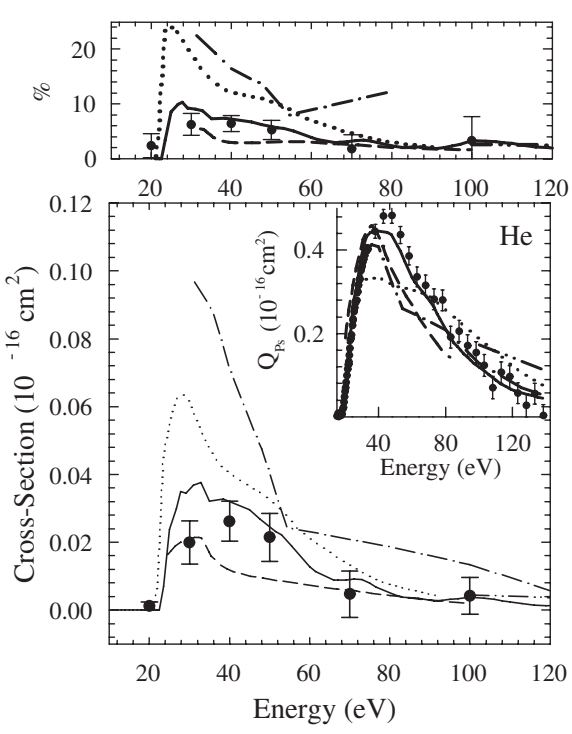

FIG. 1. The present $Q_{\mathrm{Ps}}(2 P)$ for He (circles) compared with available theories: solid curve, Ref. [19]; dotted line, Ref. [20]; chain curve, Ref. [16]; dashed curve, Ref. [17]; double chain curve, Ref. [18]. The error bars include the statistical errors on the yields and the uncertainties on the normalization factors. Above the main figure is the percentage contribution of $Q_{\mathrm{Ps}}(2 P)$ to the $Q_{\mathrm{Ps}}($ all $n)$ of Ref. [29]. In the inset, $Q_{\mathrm{Ps}}($ all $n$ ) are displayed: circles, Ref. [29]; lines, as in main figure.

Included in Figs. 1-3 are corresponding available theories. For He, they comprise the coupled-state calculation of Ref. [19], the close-coupling calculations of Refs. [17,20], the distorted-wave approach of Ref. [16], and from $100 \mathrm{eV}$, the second Born approximation of Ref. [18]. In Fig. 1, the theories exhibit a similar energy dependence to one another, all peaking at $\sim 35 \mathrm{eV}$, somewhat earlier than ex-

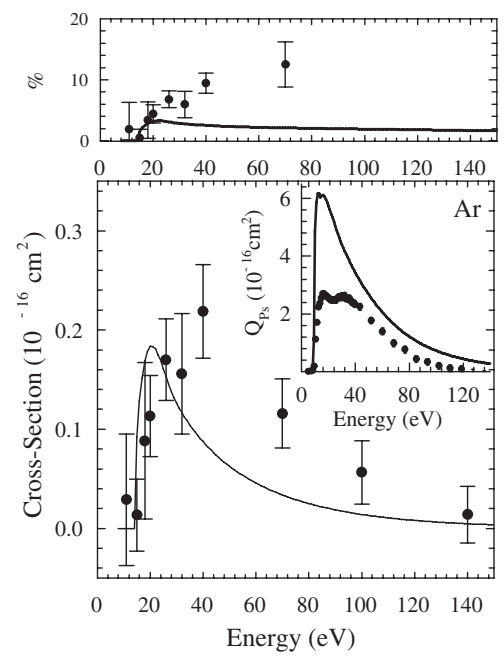

FIG. 2. The present $Q_{\mathrm{Ps}}(2 P)$ for $\mathrm{Ar}$ (circles) compared with theory [21] (line). Error bars as per Fig. 1. Above the main figure is the percentage contribution of $Q_{\mathrm{Ps}}(2 P)$ to the $Q_{\mathrm{Ps}}($ all $n)$ of Ref. [22] compared with the corresponding calculation [21,24]. In the inset, $Q_{\mathrm{Ps}}($ all $n$ ) are displayed: circles, Ref. [22]; lines, Refs. [21,24]. periment, then decreasing rapidly above $50 \mathrm{eV}$. There are, however, significant differences in absolute magnitude among them, and the best agreement is found between the present results and the elaborate calculation of Ref. [19], as was also the case between their $Q_{\mathrm{Ps}}($ all $n)$ and the most recent experimental determination [29] (see inset). The results of the close-coupling approximation calculations of Refs. [17,20] are, respectively, a factor of 2 and 3 higher in magnitude at their peak than the experimental $Q_{\mathrm{Ps}}(2 P)$.

The measurements for Ar and Xe are compared with a distorted-wave Born approximation (DWBA) calculation [21]. In Fig. 2, the theoretical results for Ar may be seen to peak earlier than experiment, undercutting it by $\sim 35 \%$ between energies of 40 and $100 \mathrm{eV}$. Finally, in the case of Xe (Fig. 3), there is a broad agreement in shape and magnitude between theory and experiment although the experimental $Q_{\mathrm{Ps}}(2 P)$ is on average higher around the peak region. Concerning $Q_{\mathrm{Ps}}($ all $n)$ for these targets, the DWBA calculation of Ref. [24] exceeds experimental measurements [22] by approximately factors of 2 and 3 (see insets).

Figure 4 compares upper and lower limit estimates of $Q_{\mathrm{Ps}}(n>1)$ [22] with the results of the DWBA and the present measurements of $Q_{\mathrm{Ps}}(2 P)$. In the case of Ar, the measured $Q_{\mathrm{Ps}}(2 P)$ accounts for approximately $1 / 3$ of the lower limit estimate of $Q_{\mathrm{Ps}}(n>1)$, while for Xe they are of a similar magnitude around the peak region.

Detection of the fluorescence of the ion (i.e., from simultaneous excitation-Ps formation or ionization) is possible above the thresholds for transitions resulting in photons of wavelengths in the range $200 \mathrm{~nm} \leq \lambda \leq 280 \mathrm{~nm}$. These thresholds, corresponding to $E_{\text {sim }}=I-6.8 \mathrm{eV}+$ $E_{\text {ex }}$ ( $I$ being the ionization energy of the ground-state target atom, $6.8 \mathrm{eV}$ the binding energy of ground-state Ps, and $E_{\mathrm{ex}}$ the relevant excitation energy of the ion), are approximately $58.6 \mathrm{eV}$ for $\mathrm{He}, 30.1 \mathrm{eV}$ for $\mathrm{Ar}$, and $21.3 \mathrm{eV}$ for

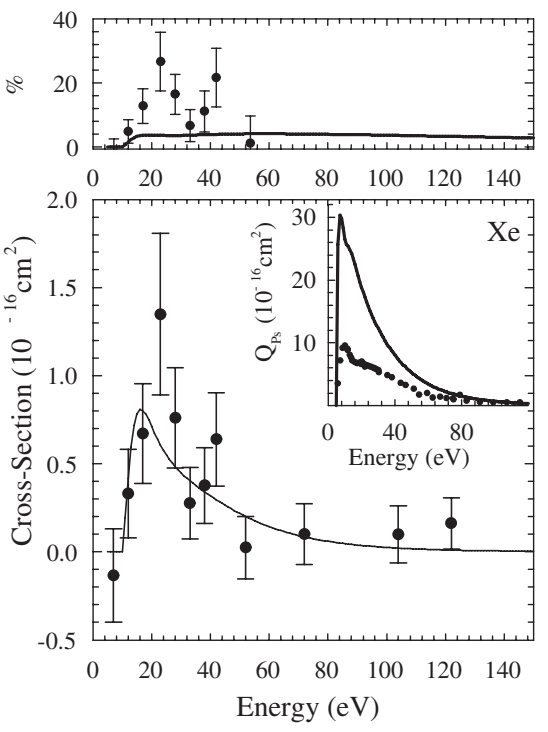

FIG. 3. As per Fig. 2 but for Xe. 

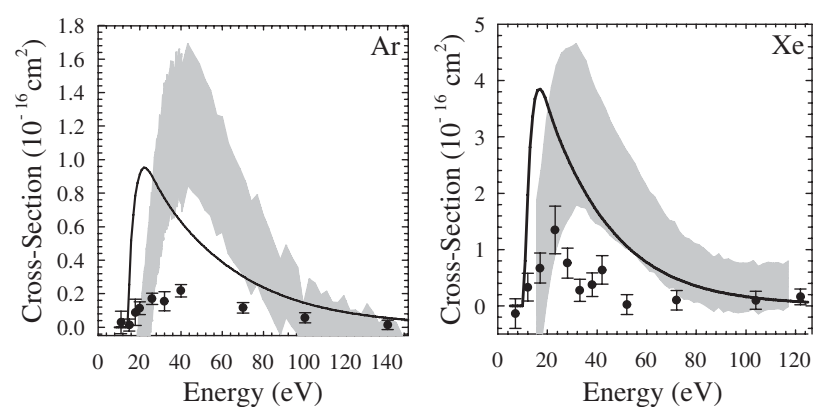

FIG. 4. Present $Q_{\mathrm{Ps}}(2 P)$ for $\mathrm{Ar}$ and Xe (circles), the limits of $Q_{\mathrm{Ps}}(n>1)$ : gray region, Ref. [22]; line, DWBA results [24].

Xe [38]. Thus, the said type of contribution, which in the case of $\mathrm{CO}_{2}$ peaks at around 4 times the threshold energy and remains significant $(\approx 30 \%$ of its maximum) at $\sim 0.5 \mathrm{keV}$ [39], may be expected to be negligible in the observed signal. In the case of Xe, explicit measurements of positron-photon coincidences (corresponding to simultaneous excitation ionization) were also performed and found to be zero within errors.

In conclusion, the first measurements of $Q_{\mathrm{Ps}}(2 P)$ have been presented for $\mathrm{He}, \mathrm{Ar}$, and $\mathrm{Xe}$ targets. The results for $\mathrm{He}$ are in good agreement with the coupled-state calculation of Ref. [19], and there is broad agreement between the experimental $Q_{\mathrm{Ps}}(2 P)$ for Xe and the DWBA of Ref. [21], while for $\mathrm{Ar}$ experiment exceeds theory. As discussed above, however, a large discrepancy exists between theory and experiment in the case of $Q_{\mathrm{Ps}}($ all $n)$ for these two targets. The contributions of $Q_{\mathrm{Ps}}(2 P)$ to the total Ps formation cross sections are found to increase from approximately $0.06 \pm 0.01$ in $\mathrm{He}$ to $0.12 \pm 0.04$ in $\mathrm{Ar}$ and $0.26 \pm 0.09$ in $\mathrm{Xe}$, in qualitative agreement with the estimates of Ref. [22].

Further attention from theory to both integral and differential state-resolved $Q_{\mathrm{Ps}}$ would seem warranted. Possible experimental investigations of metastable Ps states and molecular targets are being considered.

We thank H. R. J. Walters and C. Starrett for sending us their results prior to publication, them and A. Crowe for valuable discussions, J. Dumper and R. Jawad for technical support, and EPSRC for funding this research.

[1] A. J. Garner, G. Laricchia, and A. Ozen, J. Phys. B 29, 5961 (1996); N. Zafar et al., Phys. Rev. Lett. 76, 1595 (1996); D. E. Leslie, S. Armitage, and G. Laricchia, J. Phys. B 35, 4819 (2002); J. Beale, S. Armitage, and G. Laricchia, J. Phys. B 39, 1337 (2006); M. Skalsey et al., Phys. Rev. A 67, 022504 (2003); H. Saito and T. Hyodo, Phys. Rev. Lett. 97, 253402 (2006).

[2] S. Armitage et al., Phys. Rev. Lett. 89, 173402 (2002).

[3] S. Brawley et al., Nucl. Instrum. Methods Phys. Res., Sect. B 266, 497 (2008).

[4] D. B. Cassidy and A. P. Mills, Phys. Rev. Lett. 100, 013401 (2008).
[5] G. Gabrielse et al., Phys. Lett. B 548, 140 (2002).

[6] A. Speck et al., Phys. Lett. B 597, 257 (2004).

[7] M. H. Holzscheiter, M. Charlton, and M. M. Nieto, Phys. Rep. 402, 1 (2004).

[8] C. Starrett, M. T. McAlinden, and H. R. J. Walters, Phys. Rev. A 72, 012508 (2005).

[9] A. S. Ghosh et al., Phys. Rev. A 63, 042706 (2001).

[10] M. Barham and J. W. Darewych, J. Phys. B 41, 185001 (2008).

[11] G. Bélanger et al., Astrophys. J. 636, 275 (2006).

[12] C. Champion and C. L. Loirec, Phys. Med. Biol. 51, 1707 (2006).

[13] K. F. Canter, A. P. Mills, and S. Berko, Phys. Rev. Lett. 34, 177 (1975).

[14] G. Laricchia et al., Phys. Lett. 109A, 97 (1985).

[15] A. A. Kernoghan et al., J. Phys. B 29, 2089 (1996).

[16] P. Khan, P. S. Mazumdar, and A. S. Ghosh, Phys. Rev. A 31, 1405 (1985).

[17] R. N. Hewitt, C. J. Noble, and B. H. Bransden, J. Phys. B 25, 557 (1992).

[18] N. K. Sarkar, M. Basu, and A. S. Ghosh, Phys. Rev. A 45, 6887 (1992).

[19] C. P. Campbell et al., Nucl. Instrum. Methods Phys. Res., Sect. B 143, 41 (1998).

[20] P. Chaudhuri and S. K. Adhikari, J. Phys. B 31, 3057 (1998).

[21] S. Gilmore, Ph.D. thesis, Queen's University Belfast, 2004.

[22] G. Laricchia et al., J. Phys. B 35, 2525 (2002).

[23] M. Szłuińska, P. Van Reeth, and G. Laricchia, J. Phys. B 35, 4059 (2002).

[24] S. Gilmore, J. E. Blackwood, and H. R. J. Walters, Nucl. Instrum. Methods Phys. Res., Sect. B 221, 129 (2004).

[25] J. P. Marler, J. P. Sullivan, and C. M. Surko, Phys. Rev. A 71, 022701 (2005).

[26] L. J. M. Dunlop and G. F. Gribakin, Nucl. Instrum. Methods Phys. Res., Sect. B 247, 61 (2006).

[27] M. Charlton and G. Laricchia, J. Phys. B 23, 1045 (1990).

[28] J. Moxom, G. Laricchia, and M. Charlton, J. Phys. B 28, 1331 (1995).

[29] D. J. Murtagh et al., J. Phys. B 38, 3857 (2005).

[30] G. Laricchia et al., in Advances in Atomic, Molecular, and Optical Physics, edited by E. Arimondo, P. Berman, and C. Lin (Elsevier, New York, 2008), Vol. 56.

[31] D. J. Murtagh et al. (to be published).

[32] S. M. Curry, Phys. Rev. A 7, 447 (1973).

[33] H. R. J. W. C. Starrett and M. McAlinden, Nucl. Instrum. Methods Phys. Res., Sect. B 266, 506 (2008).

[34] C. Starrett and H. R. J. Walters, Nucl. Instrum. Methods Phys. Res., Sect. B 266, 3221 (2008).

[35] M. T. McAlinden and H. R. J. Walters, Hyperfine Interact. 89, 407 (1994).

[36] J. L. Edwards and E. W. Thomas, Phys. Rev. A 2, 2346 (1970).

[37] D. R. Bates and J.C.G. Walker, Planet. Space Sci. 14, 1367 (1966).

[38] Using National Institute of Standards and Technology Atomic Spectra Database data for the lowest energy excited ionic state radiating a photon in the sensitivity range.

[39] D. A. Cooke et al. (to be published). 